Chirurg 2009 $80: 999$

DOI 10.1007/s00104-009-1795-8

Online publiziert: 13. November 2009

๑) Springer Medizin Verlag 2009

\author{
J.R. Siewert \\ Universitätsklinikum Heidelberg
}

\section{Erst denken, dann lenken}

\section{Oberer Gastrointestinaltrakt - neoadjuvante Therapie}

Die neoadjuvante Therapie beim Karzinom des oberen Gastrointestinaltraktes ist in Anbetracht vorliegender überzeugender Daten aus großen prospektivkontrollierten Studien heute als belegt anzusehen. Dabei steht die Chemotherapie bei den Adenokarzinomen, die Radiochemotherapie bei Plattenepithelkarzinomen ganz im Vordergrund. Keine Frage ein bedeutender Fortschritt für unsere $\mathrm{Pa}$ tienten. Dieser Fortschritt darf den betroffenen Patienten heute nicht mehr vorenthalten werden, er ist „evidence based“.

Zwei wesentliche Konsequenzen ergeben sich:

- Zum einen haben wir gelernt, dass nur sogenannte „Responder“ wirklich von der neoadjuvanten Therapie profitieren. Wir müssen also alles daran setzen, den individuellen Patienten zu identifizieren, der von der neoadjuvanten Therapie profitieren wird, d.h. die neoadjuvante Therapie muss noch zielgerichteter werden. Die Rationale hinter der Suche nach dem Responder ist, dass man diese teure und aggressive Therapie den Patienten ersparen möchte, die davon voraussichtlich nicht profitieren werden. Diesen Patienten werden damit Belastungen und Enttäuschungen erspart. Des Weiteren könnten so volkswirtschaftliche Kosten eingespart werden, weil keine teuren Therapien mehr bei „zu erwartenden Nichtrespondern“ zur Anwendung kommen würden. Nicht die ohnehin kaum erreichbare Preisreduktion bei Therapieverfahren, sondern ihre gezielte Anwendung hilft Kosten im Gesundheits- system einzusparen.[BR]Dazu müssen zwei Wege erprobt, bzw. beschritten werden: Ideal wäre die Möglichkeit einer „Responsevorhersage“. Hier muss weiter nach geeigneten molekularen Markern gesucht werden. Solange derartige Marker für die klinische Routine noch nicht zur Verfügung stehen, muss auf die „Responseevaluation“ zum frühestmöglichen Zeitpunkt gesetzt werden. Die metabolische „Responseevaluation“, z. B. mittels PET-CT scheint hier zurzeit der erfolgreichste Weg zu sein.

Die andere wichtige Konsequenz heißt:

- organisatorische Voraussetzungen für die richtige Therapieentscheidung so früh wie möglich, am besten gleich nach Diagnosestellung zu schaffen. Bevor die eigentliche „Kerntherapie“ - z. B. Chirurgie - zum Einsatz kommt, muss die neoadjuvante Therapiemöglichkeit im Kreise von Experten diskutiert werden. Tumorboards sind also angezeigt! Dadurch wird die Therapie der Karzinome des oberen Gastrointestinaltraktes noch zentrumslastiger als sie ohnehin schon ist. Es führen aber viele Wege zur interdisziplinären Tumorkonferenz. Jeder muss die organisatorischen Voraussetzungen orientiert an seiner Umgebung und seinen Möglichkeiten schaffen. Darauf haben unsere Patienten Anspruch.

Deshalb heißt das Motto der modernen onkologischen Therapie der Tumoren des oberen Gastrointestinaltraktes: Erst den- ken - im Tumorboard -, dann lenken, d.h. den Patienten nach interdisziplinärer Tumorentscheidung der adäquaten Therapie, in der Regel der adäquaten multimodalen Therapie zuzuführen.

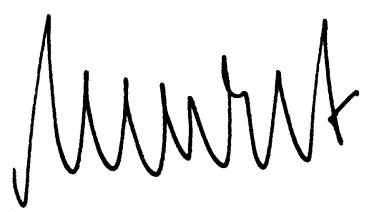

Prof. Dr. Dr. h.c. J.R. Siewert

Korrespondenzadresse

Prof. Dr. Dr. h.c. J.R. Siewert

Universitätsklinikum Heidelberg, Im Neuenheimer Feld 672, 69120 Heidelberg

JR.Siewert@med.uni-heidelberg.de 